\title{
Phase 2 study of axicabtagene ciloleucel in Japanese patients with relapsed or refractory large B-cell lymphoma
}

\author{
Koji Kato $^{1}$ (1) Shinichi Makita ${ }^{2} \cdot$ Hideki Goto $^{3} \cdot$ Junya Kanda $^{4} \cdot$ Nobuharu Fujii $^{5} \cdot K_{\text {Kazuyuki Shimada }} \cdot$ Koichi Akashi $^{1}$. \\ Koji Izutsu $^{2} \cdot$ Takanori Teshima $^{3} \cdot$ Natsuko Fukuda $^{7} \cdot$ Tokuhito Sumitani $^{7} \cdot$ Hiroyuki Sumi $^{7} \cdot$ Shinji Shimizu $^{7}$. \\ Yasuyuki Kakurai $^{7} \cdot$ Kenji Yoshikawa $^{7} \cdot$ Kensei Tobinai $^{8} \cdot$ Noriko Usui $^{9} \cdot$ Kiyohiko Hatake $^{10}$
}

Received: 30 June 2021 / Accepted: 15 September 2021 / Published online: 1 October 2021

(c) The Author(s) 2021

\begin{abstract}
Background Axicabtagene ciloleucel (axi-cel) is an autologous chimeric antigen receptor T-cell based anti-CD19 therapy. The ZUMA-1 study, multicenter, single-arm, registrational Phase 1/2 study of axi-cel demonstrated high objective response rate in patients with relapsed/refractory large B-cell lymphoma. Here, we present the results of the bridging study to evaluate the efficacy and safety of axi-cel in Japanese patients (JapicCTI-183914).

Methods This study was the phase 2, multicenter, open-label, single-arm trial. Following leukapheresis, axi-cel manufacturing and lymphodepleting chemotherapy, patients received a single infusion of axi-cel $\left(2.0 \times 10^{6} \mathrm{cell} / \mathrm{sg}\right)$. Bridging therapy between leukapheresis and conditioning chemotherapy was not allowed. The primary endpoint was objective response rate. Results Among 17 enrolled patients, 16 received axi-cel infusion. In the 15 efficacy evaluable patients, objective response rate was $86.7 \%$ (95\% confidence interval: 59.5-98.3\%); complete response/partial response were observed in 4 (26.7\%)/9 $(60.0 \%)$ patients, respectively. No dose-limiting toxicities were observed. Grade $\geq 3$ treatment-emergent adverse events occurred in $16(100 \%)$ patients-most commonly neutropenia (81.3\%), lymphopenia (81.3\%) and thrombocytopenia (62.5\%). Cytokine release syndrome occurred in $13(81.3 \%)$ patients (12 cases of grade 1 or 2 and 1 case of grade 4 ). No neurologic events occurred. Two patients died due to disease progression, but no treatment-related death was observed by the data-cutoff date (October 23, 2019).
\end{abstract}

Conclusion The efficacy and safety of axi-cel was confirmed in Japanese patients with relapsed/refractory large B-cell lymphoma who have otherwise limited treatment options.

Trial registration JapicCTI-183914.

Keywords Axicabtagene ciloleucel $\cdot$ CD19-specific chimeric antigen receptor $\cdot$ Japan $\cdot$ KTE-C19 $\cdot$ Non-Hodgkin lymphoma

\section{Introduction}

Non-Hodgkin lymphoma (NHL) is the tenth most common type of cancer in Japan and diffuse large B-cell lymphoma (DLBCL) is the most common subtype [1,2]. Although the combination of rituximab, cyclophosphamide, doxorubicin, vincristine, and prednisone (R-CHOP) has improved the prognosis of DLBCL patients, $30-50 \%$ of them are not cured by this treatment [3]. SCHOLAR-1, a pooled retrospective analysis of patients with refractory DLBCL showed

Koji Kato

kato.koji.429@m.kyushu-u.ac.jp

Extended author information available on the last page of the article a median overall survival (OS) of 6.3 months and an objective response rate (ORR) of $26 \%$ [4]. Axicabtagene ciloleucel (axi-cel, KTE-C19) is one of the autologous chimeric antigen receptor (CAR) T-cell therapies targeting CD19 which is considered as an optimal therapeutic target due to its uniform expression on malignant B-cells [5]. While axicel employs CD28 as a costimulatory domain [6], other antiCD19 CAR T cells such as tisagenlecleucel (tisa-cel) [7] and lisocabtagene maraleucel (liso-cel) [8] employ 4-1BB as a costimulatory domain. ZUMA-1 (NCT02348216), multicenter, international (not including Japan), pivotal phase 1/2, single-arm study of axi-cel, demonstrated $82 \%$ of ORR, $54 \%$ of complete response (CR) rate, and 25.8 months of median 
OS in the relapsed/refractory (R/R) large B-cell lymphoma patients [6, 9-11].

To confirm the efficacy and safety of axi-cel in Japanese patients, J201 bridging study was conducted (JapicCTI-183914). In this paper, results of this bridging study are reported.

\section{Patients and methods}

\section{Study design}

This was a phase 2, multicenter, open-label, single-arm study to evaluate the efficacy and safety of axi-cel in Japanese patients with R/R large B-cell lymphoma.

Patients meeting the eligibility criteria were enrolled and mononuclear cells were obtained by leukapheresis at the study site. The leukapheresed cells were shipped to the manufacturing site and processed to manufacture axi-cel, which was cryopreserved and shipped back to the study site. Bridging chemotherapy to control lymphoma between leukapheresis and conditioning chemotherapy was not permitted. Cyclophosphamide $500 \mathrm{mg} / \mathrm{m}^{2} /$ day and fludarabine $30 \mathrm{mg} /$ $\mathrm{m}^{2} /$ day were administered for 3 consecutive days $(-5,-4$, and -3 ) before axi-cel infusion as conditioning chemotherapy (also known as lymphodepleting chemotherapy; see supplementary materials for details). The number of cells was adjusted to the target dose of anti-CD19 CAR T cells $\left(2.0 \times 10^{6}\right.$ cells $/ \mathrm{kg}$ of body weight) (Fig. 1$)$.
This study comprised stages 1 and 2 (Fig. S1). An interim analysis assessing ORR and safety was planned for early efficacy evaluation and early futility termination. The interim analysis was to be conducted with ten patients. If the drug was not deemed effective nor ineffective in the interim analysis, six patients were planned to be added. Since this is the first study in Japanese patients, the tolerability of axi-cel was also assessed once dose-limiting toxicity (DLT) evaluation period (28 days) for the first three subjects was finished (see supplementary materials).

The study protocol was approved by the independent ethics committees or institutional review boards of the study sites, and the study was conducted in accordance with the principles of the Declaration of Helsinki, International Conference on Harmonisation-Good Clinical Practice, and other applicable regulatory requirements. All patients provided written informed consent.

\section{Patients}

Key inclusion criteria included the following: age $\geq 20$ years; Eastern Cooperative Oncology Group performance status (ECOG PS) 0/1; histologically confirmed aggressive B-cell NHL (including DLBCL, Primary mediastinal large B-cell lymphoma (PMBCL), transformed follicular lymphoma (TFL), High-grade B-cell lymphoma (HGBCL) with $M Y C$ and $B C L 2$ and/or BCL6 rearrangement; or HGBCL not otherwise specified) as defined by the World Health Organization (WHO) 2016 criteria [12]; chemorefractory

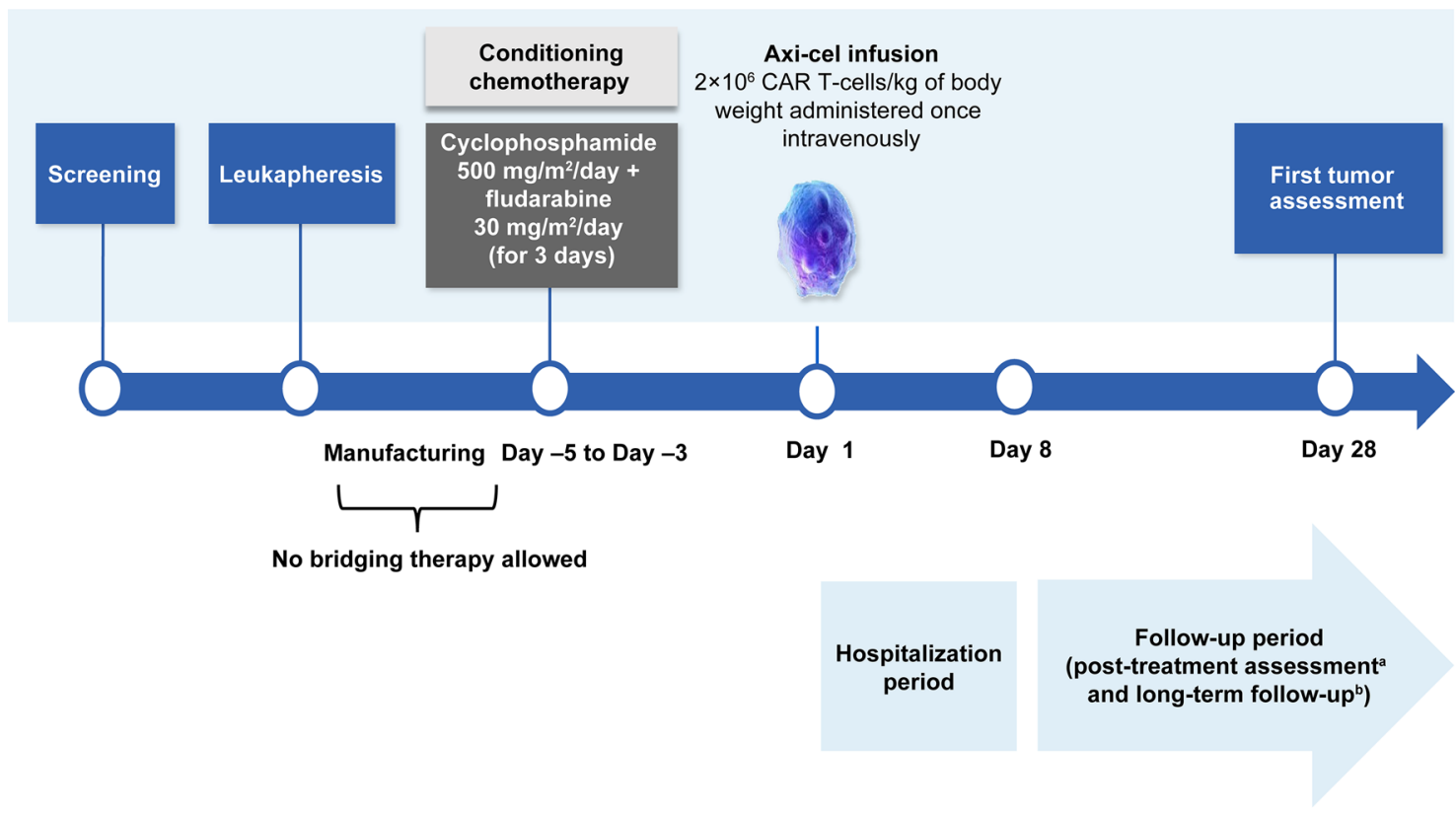

Fig. 1 Study design. ${ }^{a}$ Week 2 , week 4 , month 2, and month 3 . bevery 3 months \pm 2 weeks to month 18 , every $6 \pm 1$ months from month 24 to month 60 , and visit once a year \pm 3 months from years 6-15. axi-cel axicabtagene ciloleucel, CAR chimeric antigen receptor 
disease or relapse $\leq 12$ months after autologous stem cell transplantation (ASCT); prior use of an anti-CD20 monoclonal antibody and anthracycline-containing chemotherapy or prior chemotherapy for follicular lymphoma in patients with TFL; no evidence of central nervous system (CNS) lymphoma; absolute neutrophil count $\geq 1000 / \mu \mathrm{L}$; platelet count $\geq 75,000 / \mu \mathrm{L}$; and absolute lymphocyte count $\geq 100$ / $\mu \mathrm{L}$.

Key exclusion criteria included a history of malignancy within the past 3 years, prior allogeneic stem cell transplantation, prior CD19-targeted therapy, prior CAR T-cell therapy or other genetically modified T-cell therapy.

\section{Endpoints}

Disease response was evaluated per the International Working Group (IWG) 2007 criteria [13]. The primary efficacy endpoint was ORR (defined as the proportion of patients who achieved CR or PR) based on investigator assessment. Secondary endpoints included ORR based on central diagnostic imaging evaluation (see supplementary materials); best response observed among all disease assessments by an investigator (defined in the order of CR, PR, stable disease [SD], progressive disease [PD], and not evaluable); CR rate; duration of response (DOR); time to response (TTR); progression-free survival (PFS); and OS. Pharmacokinetic endpoints such as the concentration of axi-cel in blood and safety endpoints such as DLTs, treatment-emergent adverse events (TEAEs), serious adverse events (SAEs), and adverse events (AEs) of special interest were also evaluated. TEAEs were graded according to the National Cancer Institute Common Terminology Criteria for Adverse Events (CTCAE) version 4.03 [14]. Cytokine release syndrome (CRS) was graded according to the criteria of Lee et al. [15].

\section{Statistical analysis}

Considering the ORR was 26\% in SCHOLAR-1 [4] and $82 \%$ in ZUMA-1 [6], the threshold ORR was selected as $26 \%$, and the expected ORR was set at $60 \%$. The study design was decided with reference to the two-stage designs optimal under the alternative hypothesis suggested by Mander and Thompson [16], with a one-sided significance level of 5\% and power of $\geq 80 \%$. Analyses were performed using SAS ${ }^{\circledR}$ version 9.4 (SAS Institute Inc., Cary, NC, USA) and Phoenix WinNonlin version 8.1 (Certara G.K., Princeton, NJ, USA). ORR with exact two-sided $90 \%$ confidence interval (CI) and two-sided 95\% CI based on the Clopper-Pearson method were calculated. The safety analysis set included all patients who received axi-cel infusion. The pharmacokinetic (PK) analysis set included patients in the safety analysis set who had $\geq 1$ available PK data. The modified intent-to-treat (mITT) analysis set included patients who received axi-cel infusion at a dose of $\geq 1.0 \times 10^{6} \mathrm{CAR} \mathrm{T}$ cells $/ \mathrm{kg}$. The primary efficacy analysis set included patients in the mITT analysis set with primary endpoint data. The interim analysis set included the first ten treated patients from the primary efficacy analysis set.

\section{Results}

\section{Patient disposition}

The data cutoff date for the interim analysis was July 15 , 2019 and that for the updated analysis was October 23, 2019. At data cutoff for the updated analysis, 20 patients had provided informed consent, of whom three patients did not meet the eligibility criteria and were excluded. Seventeen patients were enrolled in the study, and one patient underwent leukapheresis but discontinued the study before receiving conditioning chemotherapy due to disease progression. Sixteen patients received axi-cel and had the opportunity to be followed for a minimum of 3.0 months, with a median (range) actual follow-up time of 5.5 (3.0-10.4) months. In one patient, infusion was discontinued due to an anaphylactic reaction (considered as a reaction to dimethyl sulfoxide). This patient was included in the safety analysis set but excluded from the mITT analysis set due to an axi-cel infusion dose $<1.0 \times 10^{6}$ cells $/ \mathrm{kg}$. Two patients died due to disease progression during the study and 14 patients remained on study (Fig. 2).

\section{Manufacturing and administration of axi-cel}

The median (range) time from leukapheresis to the receipt of axi-cel at the study site was $26.5(23-38)$ days $(n=16)$. The median (range) time from leukapheresis to axi-cel infusion was 29 (25-48) days. A total of 15 patients received the target dose of axi-cel $\left(2.00 \times 10^{6}\right.$ cells $\left./ \mathrm{kg}\right)$; one patient with the anaphylactic reaction received $0.4 \times 10^{6}$ cells $/ \mathrm{kg}$ of axi-cel. Axi-cel was manufactured in the United States and shipped and administered to patients in Japan.

\section{Patient demographics and baseline clinical characteristics}

Of the 16 patients who received axi-cel, 14 (87.5\%) were diagnosed with DLBCL per investigator assessment. The median (range) age was 58 (44-70) years, and five (31.3\%) patients aged $\geq 65$ years were included. Seven (43.8\%) patients had stage IV disease. At baseline, 13 (81.3\%) patients were CD19 positive and 1 patient was negative by immunohistochemistry (data were missing for 2 patients). Twelve (75.0\%) patients had received $\geq 3$ prior therapies, $10(62.5 \%)$ patients were refractory to second- or 


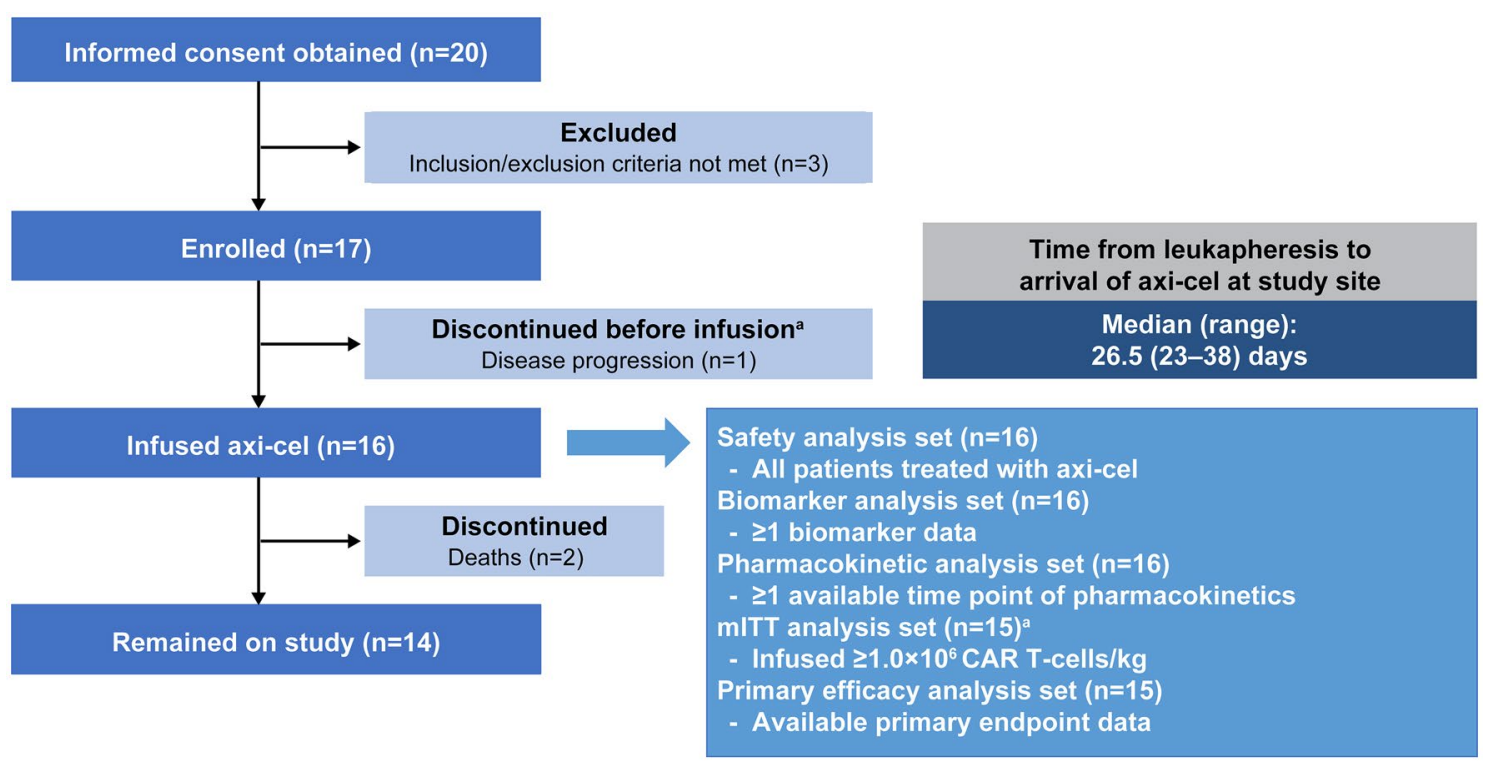

Fig. 2 Patient disposition. anfusion was discontinued due to an anaphylactic reaction in one patient. This patient was excluded from the mITT analysis set because the infused axi-cel dose was $<1.0 \times 10^{6}$

subsequent-line therapy; and $6(37.5 \%)$ patients relapsed after ASCT within a year (Table 1).

\section{Efficacy}

In the interim analysis, among the first 10 treated patients in the primary efficacy analysis set, CR or PR was observed in 9 patients and the prespecified criteria for efficacy was met. By the time the treatment was found effective in interim analysis, 17 patients had been already enrolled and 16 patients were treated consequently. In subsequent sections, data of the updated analysis for 16 patients with a minimum follow-up of 3.0 months (data cutoff: October 23, 2019) are described.

ORR based on investigator assessment in the primary efficacy analysis set was 86.7\% (95\% CI 59.5-98.3\%; 13/15 patients) (Table 2). The best response was CR in 4 (26.7\%) patients, PR in $9(60.0 \%)$ patients, and SD and PD in 1 (6.7\%) patient each (Table 2). The individual responses at each timepoint are shown in Table S1. The ORR by central imaging evaluation was $60.0 \%$ (95\% CI 32.3-83.7\%; 9/15 patients) with CR in four (26.7\%) patients. Two patients were judged as not evaluable in central assessment. And the selected lesions for disease assessment were different from those selected in investigator assessment in some cases.

The median DOR (95\% CI) was 5.6 (2.2-not estimable) months. Among 13 patients who responded to axi-cel, 8 (61.5\%) showed an ongoing response (Fig. 3a). The median (range) TTR and time to CR were $0.95(0.85-2.86)$ and 0.97 (0.95-1.05) months, respectively. Pseudo-progression was cells $/ \mathrm{kg}$ of body weight. axi-cel axicabtagene ciloleucel, CAR chimeric antigen receptor, $m I T T$ modified intent-to-treat

not observed. The median PFS (95\% CI) was 6.5 (2.9-not estimable) months. Eight (53.3\%) patients did not meet the criteria for disease progression or death (Fig. 3b). The median OS (95\% CI) was not reached (6.9-not estimable), and 13 patients $(86.7 \%)$ were alive at data cutoff (Fig. 3c).

\section{Safety}

DLT was assessed with the first three enrolled patients and no DLT was observed. Grade $\geq 3$ TEAEs occurred in 16 (100\%) patients (Table S2). TEAEs related to conditioning chemotherapy and TEAEs related to axi-cel occurred in $16(100 \%)$ patients each and were all grade $\geq 3$. A TEAE (anaphylactic reaction) leading to discontinuation of axicel occurred in one (6.3\%) patient with a history of infusion reaction to rituximab. Serious TEAEs occurred in 13 $(81.3 \%)$ patients. No fatal TEAEs was reported. The most common TEAEs of any grade occurring in $\geq 8$ patients were pyrexia (87.5\%), lymphopenia $(81.3 \%)$, neutropenia (81.3\%), thrombocytopenia (75.0\%), leukopenia (56.3\%), decreased appetite (56.3\%), diarrhea and nausea (50\% each) (Table 3), while the most common grade $\geq 3$ TEAEs that occurred in $\geq 8$ patients were lymphopenia ( $81.3 \%$ ), neutropenia $(81.3 \%)$, thrombocytopenia (62.5\%), and leukopenia $(56.3 \%)$ (Table 3).

Treatment-emergent CRS was reported in 13 (81.3\%) patients (12 cases of grade 1 or 2 and 1 case of grade 4 ). Eleven CRS cases were considered as SAEs. The most common symptoms of CRS were pyrexia $(81.3 \%)$, diarrhea $(18.8 \%)$, hypotension $(12.5 \%)$, and hypoxia $(12.5 \%)$ 
Table 1 Patient demographics and baseline clinical characteristics (safety analysis set, $n=16$ )
Safety analysis set $(n=16)$

Disease type by investigator, $n(\%)$

DLBCL

$14(87.5)^{\mathrm{a}}$

PMBCL

$1(6.3)$

TFL

$1(6.3)$

HGBCL

$0(0.0)$

Age

Median (range), years

$58(44-70)$

$\geq 65$ years, $n(\%)$

5 (31.3)

Sex, $n(\%)$

Male

$11(68.8)$

Female

5 (31.3)

Body weight, median (range), $\mathrm{kg}$

$63.6(44.6-78.2)$

ECOG PS, $n(\%)$

0

$12(75.0)$

1

4 (25.0)

Disease stage at study entry ${ }^{\mathrm{b}}, n(\%)$

I

4 (25.0)

II

$4(25.0)$

III

$1(6.3)$

IV

$7(43.8)$

Bulky disease ( $\geq 1$ lesion of $10 \mathrm{~cm}$ in diameter), $n(\%)$

Yes

$1(6.3)$

No

$15(93.8)$

Tumor burden (SPD) for target lesions, $\mathrm{mm}^{2}$

Mean (SD)

$4544.9(6748.17)$

Median (range)

$1991.5(288-26,360)$

International Prognostic Index, $n(\%)$

0

$3(18.8)$

$4(25.0)$

$3(18.8)$

$4(25.0)$

2 (12.5)

$0(0.0)$

CD19 positivity at baseline, $n(\%)$

Yes

$13(81.3)$

No

$1(6.3)$

$2(12.5)$

Missing

$12(75.0)$

$\geq 3$ lines of prior chemotherapy, $n(\%)$

$0(0.0)$

Primary refractory

$10(62.5)$

Refractory to second- or subsequent-line therapy

$6(37.5)$

Relapse after ASCT

Baseline value is defined as the last value taken before conditioning chemotherapy

$A S C T$ autologous stem cell transplantation, $C D$ cluster of differentiation, $D L B C L$ diffuse large B-cell lymphoma, ECOG PS Eastern Cooperative Oncology Group performance status, $H G B C L$ high-grade B-cell lymphoma, $P M B C L$ primary mediastinal large B-cell lymphoma, $S D$ standard deviation, $S P D$ sum of the products of the greatest diameters, TFL transformed follicular lymphoma

${ }^{a}$ One patient was confirmed as having HGBCL by central read assessment

${ }^{\mathrm{b}}$ Cotswolds modification of the Ann Arbor staging system 
Table 2 ORR and best response based on investigator/assessment (primary efficacy analysis set; $n=15$ )

\begin{tabular}{llr}
\hline & $n(\%)$ & $95 \% \mathrm{CI}^{\mathrm{a}}$ \\
\hline ORR $^{\mathrm{b}}: \mathrm{CR}+\mathrm{PR}$ & $13(86.7)$ & $59.5-98.3$ \\
Best response & \\
$\mathrm{CR}$ & $4(26.7)$ & $7.8-55.1$ \\
$\mathrm{PR}$ & $9(60.0)$ & $32.3-83.7$ \\
$\mathrm{SD}$ & $1(6.7)$ & $0.2-31.9$ \\
$\mathrm{PD}$ & $1(6.7)$ & $0.2-31.9$ \\
Not evaluable & $0(0.0)$ & $0.0-21.8$ \\
\hline
\end{tabular}

$C I$ confidence interval, $C R$ complete response, $I W G$ International Working Group, $O R R$ objective response rate, $P D$ progressive disease, $P R$ partial response, $S D$ stable disease

${ }^{a}$ Based on the Clopper-Pearson method

${ }^{\mathrm{b}}$ Based on investigator assessment per the IWG 2007 criteria

(Table S3). The median (range) duration from axi-cel infusion to first CRS event was $2.0(1-11)$ days, and the median time to resolution was 16.5 days. Tocilizumab was administered in $11(68.8 \%)$ patients. Steroids were administered in nine $(56.3 \%$ ) patients (for details of $\mathrm{AE}$ management, see Tables S4 and S5). No neurologic events or tumor lysis syndromes (TLSs) were reported. Among late-onset cytopenias (defined as any cytopenias present on or after 30 days), thrombocytopenia occurred in 12 (75.0\%), neutropenia in $11(68.8 \%)$, anemia in $5(31.3 \%)$, and febrile neutropenia in $2(12.5 \%)$ patients (Table 4$)$. Among prolonged cytopenias (defined as any cytopenias with duration $\geq 30$ days or consecutive events with a combined duration $\geq 30$ days), neutropenia occurred in three $(18.8 \%)$ patients; thrombocytopenia and anemia occurred in one $(6.3 \%)$ patient each (Table 4$)$. Three grade $\geq 3$ infections were reported in two patients, with abdominal infection reported in one patient and acute sinusitis and infection reported in another patient $(n=1 ; 6.3 \%$ each); these events occurred more than 30 days after axi-cel infusion and resolved with medication and hospitalization or medication alone.

The proportion of patients with detectable B cells in blood was $37.5 \%$ (6/16 patients) at baseline (prior to conditioning chemotherapy), $6.3 \%$ (1/16 patients) at week $4,0 \%$ (0/15 patients) at month 3 . Hypogammaglobulinemia occurred in three $(18.8 \%)$ patients (grade $\geq 3$ : two [12.5\%] patients), and all were treated with immunoglobulins.

No incidence or exacerbation of autoimmune disease or secondary malignancies was reported. All patients tested negative for antibody to FMC63 (the parental murine antibody used for development of the anti-CD19 single-chain variable fragment region of the CAR construct) and replication-competent retrovirus.

\section{CAR T cell expansion}

Median anti-CD19 CAR T cell level in blood reached the maximum at 14 days after axi-cel infusion and decreased toward baseline (Fig. 4). The median (range) $\mathrm{T}_{\max }$ for antiCD19 CAR T cells in the blood, maximum blood concentration $\left(\mathrm{C}_{\max }\right)$, and area under the CAR T cells in blood-time curve up to 28 days $\left(\mathrm{AUC}_{28 \mathrm{~d}}\right)$ were 11 (7-28) days, 12.7 (0.9-297.4) cells/ $\mu \mathrm{L}$, and $187.5(16.9-4244.5)$ cells $\times$ days/ $\mu \mathrm{L}$, respectively. At 6 months, CAR T cells were measurable in the blood for $6 / 7$ evaluable patients. There was no clear correlation between CAR T cell expansion (AUC and peak level) and efficacy or severity of CRS in this study.

\section{Outcome of axi-cel re-administration}

Axi-cel was re-administered in one patient whose clinical course before re-administration was CR at week 4 and month 3 and PD at month 6. CD19 expression on relapsed lymphoma tissue was confirmed by immunohistochemistry. Axi-cel manufactured for the first administration had been partially cryo-preserved as a second bag and it was re-administered on day 260 . The pharmacokinetic parameters $\left(\mathrm{T}_{\max }, \mathrm{C}_{\max }\right.$, and $\left.\mathrm{AUC}_{28 \mathrm{~d}}\right)$ after re-administration were not significantly different from those for the first dose. The best response after re-administration was $\mathrm{CR}$ at week 4 . The profile of TEAEs after re-administration was consistent with that reported for the main analysis.

\section{Discussion}

This is the first study evaluating the efficacy, safety, and pharmacokinetics of axi-cel in Japanese patients. The primary endpoint ORR was $86.7 \%$ which is comparable with that in ZUMA-1 [6, 10], and higher than the historical data of SCHOLAR-1 (ORR 26\%) [4]. Considering the patient baseline characteristics (e.g., 12 patients had received $\geq 3$ prior lines of chemotherapy, and 6 patients relapsed within 12 months after ASCT), this ORR is encouraging. The CR rate in this study was lower than that in ZUMA-1 [6, 10], which can mainly be attributed to the shorter followup period. Interpretations of DOR and PFS are currently limited due to short observation period. Regarding CAR T cell expansion, median $\mathrm{AUC}_{28 \mathrm{~d}}$ and median $\mathrm{C}_{\max }$ levels were lower than those in ZUMA-1 but were within the range of variation in ZUMA-1 [6].

Finding biomarkers that can predict efficacy of axi-cel is important for patient stratification. Patients characteristic and/or product characteristics have been studied using ZUMA-1 data $[17,18]$. It was suggested that low tumor 
Fig. 3 Kaplan-Meier estimates for (a) DOR (primary efficacy analysis set), (b) PFS (primary efficacy analysis set), and (c) OS (mITT analysis set).

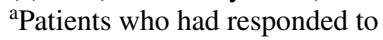
axi-cel in the primary efficacy analysis set. axi-cel axicabtagene ciloleucel, DOR duration of response, IITT modified intent-to-treat; $O S$ overall survival, $P F S$ progression-free survival a

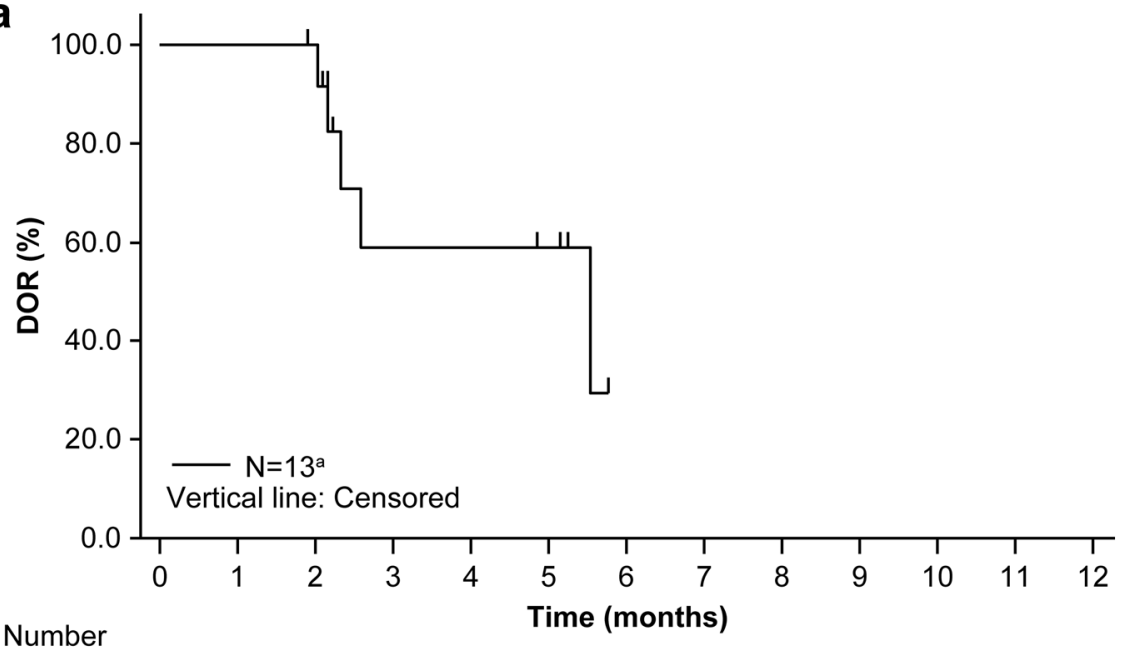

Number

$\begin{array}{lllllll}\text { at risk } & 13 & 13 & 12 & 5 & 5 & 4\end{array}$

b

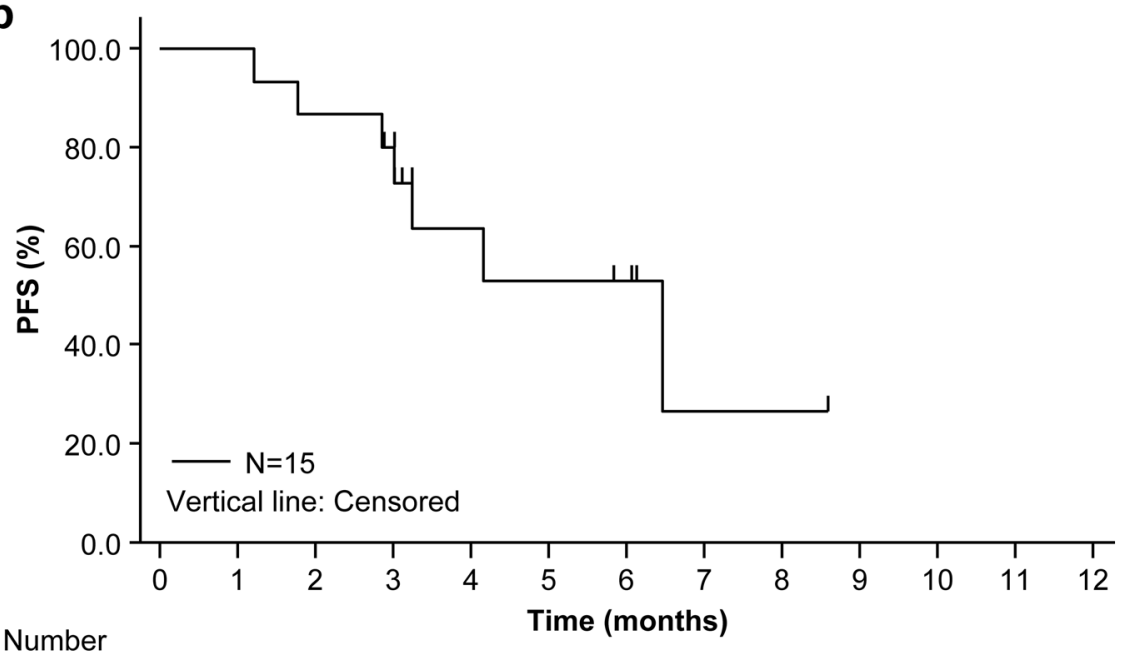

C

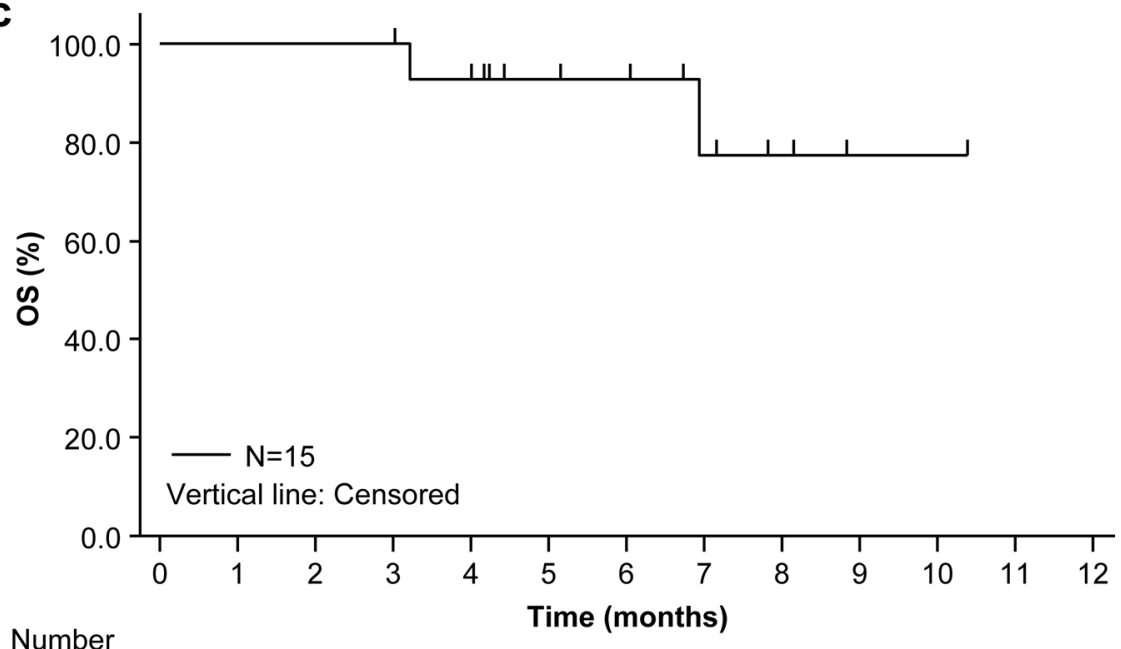

Number

15

15

13

8

3

1 
Table 3 TEAEs by CTCAE grade (safety analysis set; $n=16$ )

\begin{tabular}{|c|c|c|}
\hline Preferred term ${ }^{\mathrm{a}}$ & All grade, $n(\%)$ & Grade $\geq 3, n(\%)$ \\
\hline Pyrexia & $14(87.5)$ & $2(12.5)$ \\
\hline Lymphopenia $^{\mathrm{b}}$ & $13(81.3)$ & $13(81.3)$ \\
\hline Neutropenia $^{c}$ & $13(81.3)$ & $13(81.3)$ \\
\hline Thrombocytopenia $^{\mathrm{d}}$ & $12(75.0)$ & $10(62.5)$ \\
\hline Leukopenia $^{\mathrm{e}}$ & $9(56.3)$ & $9(56.3)$ \\
\hline Decreased appetite & $9(56.3)$ & $4(25.0)$ \\
\hline Diarrhea & $8(50.0)$ & $3(18.8)$ \\
\hline Nausea & $8(50.0)$ & $0(0.0)$ \\
\hline Febrile neutropenia & $7(43.8)$ & $7(43.8)$ \\
\hline Anemia & $7(43.8)$ & $5(31.3)$ \\
\hline $\begin{array}{l}\text { Alanine aminotransferase } \\
\text { increased }\end{array}$ & $7(43.8)$ & $1(6.3)$ \\
\hline $\begin{array}{l}\text { Aspartate aminotransferase } \\
\text { increased }\end{array}$ & $7(43.8)$ & $1(6.3)$ \\
\hline Malaise & $6(37.5)$ & $0(0.0)$ \\
\hline Headache & $5(31.3)$ & $0(0.0)$ \\
\hline Hypoxia & $4(25.0)$ & $1(6.3)$ \\
\hline Hypophosphatemia & $3(18.8)$ & $3(18.8)$ \\
\hline Hypogammaglobulinemia & $3(18.8)$ & $2(12.5)$ \\
\hline Hyponatremia & $3(18.8)$ & $1(6.3)$ \\
\hline Hypotension & $3(18.8)$ & $1(6.3)$ \\
\hline Vomiting & $3(18.8)$ & $0(0.0)$ \\
\hline Upper respiratory tract infection & $3(18.8)$ & $0(0.0)$ \\
\hline Insomnia & $3(18.8)$ & $0(0.0)$ \\
\hline Nasopharyngitis & $3(18.8)$ & $0(0.0)$ \\
\hline
\end{tabular}

CTCAE Version 4.03. TEAEs (all grade) that occurred in $\geq 3$ patients are listed

CTCAE Common Terminology Criteria for Adverse Events, MedDRA Medical Dictionary for Regulatory Activities, TEAE treatment-emergent adverse event

${ }^{\mathrm{a}}$ Coded per MedDRA version 21.0

${ }^{b}$ Lymphopenia includes lymphopenia and lymphocyte count decreased

${ }^{\mathrm{c}}$ Neutropenia includes neutropenia and neutrophil count decreased

${ }^{\mathrm{d}}$ Thrombocytopenia includes thrombocytopenia and platelet count decreased

${ }^{\mathrm{e}}$ Leukopenia includes leukopenia and white blood cell count decreased

burden, low systemic inflammation, and high product $\mathrm{CCR} 7^{+} \mathrm{CD} 45 \mathrm{RA}^{+} \mathrm{T}$ cells were associated with durable responses [17].

No additional safety concerns were raised and no DLTs were observed in Japanese patients. The occurrence of common TEAEs, serious TEAEs, and AEs of special interest in this study was consistent with that in ZUMA-1 $[6,10]$. CRS and immune effector cell-associated neurotoxicity syndrome are most frequently associated with CAR T-cell therapy and administration of tocilizumab, an anti-interleukin IL-6 receptor antibody, with or without
Table 4 Late-onset and prolonged cytopenias (safety analysis set; $n=16$ )

\begin{tabular}{lcc}
\hline $\begin{array}{l}\text { Adverse event } \\
\text { Preferred term }\end{array}$ & Any grade, $n(\%)$ & Grade $\geq 3, n(\%)$ \\
\hline Late-onset cytopenias $^{\mathrm{a}}$ & & \\
Thrombocytopenia $^{\mathrm{b}}$ & $12(75.0)$ & $10(62.5)$ \\
Neutropenia $^{\mathrm{c}}$ & $11(68.8)$ & $11(68.8)$ \\
Anemia & $5(31.3)$ & $4(25.0)$ \\
Febrile neutropenia $_{\text {Prolonged cytopenias }}^{\mathrm{d}}$ & $2(12.5)$ & $2(12.5)$ \\
Neutropenia $^{\mathrm{c}}$ & & $3(18.8)$ \\
Thrombocytopenia $^{\mathrm{b}}$ & $1(6.3)$ & $0(0.0)$ \\
Anemia & $1(6.3)$ & $0(0.0)$ \\
\hline
\end{tabular}

Coded with MedDRA version 21.0

axi-cel axicabtagene ciloleucel, MedDRA Medical Dictionary for Regulatory Activities

${ }^{a}$ Late-onset cytopenias were defined as any cytopenias present on or after 30 days of axi-cel infusion, including those that started after 30 days from axi-cel infusion and those that started earlier but were ongoing on or after 30 days of axi-cel infusion

${ }^{\mathrm{b}}$ Thrombocytopenia includes thrombocytopenia and platelet count decreased

${ }^{\mathrm{c}}$ Neutropenia includes neutropenia and neutrophil count decreased

${ }^{\mathrm{d}}$ Prolonged cytopenias were defined as any cytopenias with duration $\geq 30$ days or any consecutive events of cytopenias with a combined duration $\geq 30$ days

steroids has been found to be effective in reversing CRS [19]. In this study, CRS occurred in 13 patients (1 patient of grade $\geq 3$ ), and many of the events were managed with tocilizumab and steroid. No neurologic events occurred, the reason for which is however unknown. A real world data with large population will be necessary for further investigation.

Late-onset and prolonged cytopenias, other common adverse events related to CAR T-cell therapy [19], were observed in this study as well. A similar phenomenon was reported in a different study with a different product [20]. In the 24 months analysis of ZUMA-1, less than $20 \%$ of the patients had Grade $\geq 3$ cytopenia at month 3 and beyond suggesting a gradual recovery of those cytopenias [10]. The proportion of patients with detectable B cells in blood was $37.5 \%$ at baseline, and $0 \%$ at month 3 . In the ZUMA- 1 study, B-cell recovery was observed in $75 \%$ of patients with an ongoing response at 24 months [10]. Future follow-up is necessary to ascertain the level of B-cell recovery in Japanese patients. Three grade $\geq 3$ infections were reported more than 30 days after axi-cel infusion but all of them resolved with treatment.

Timely delivery is one of the key success factors for CAR T-cell therapy. Axi-cel arrived at study sites with the median time from leukapheresis to the receipt of 26.5 days. 


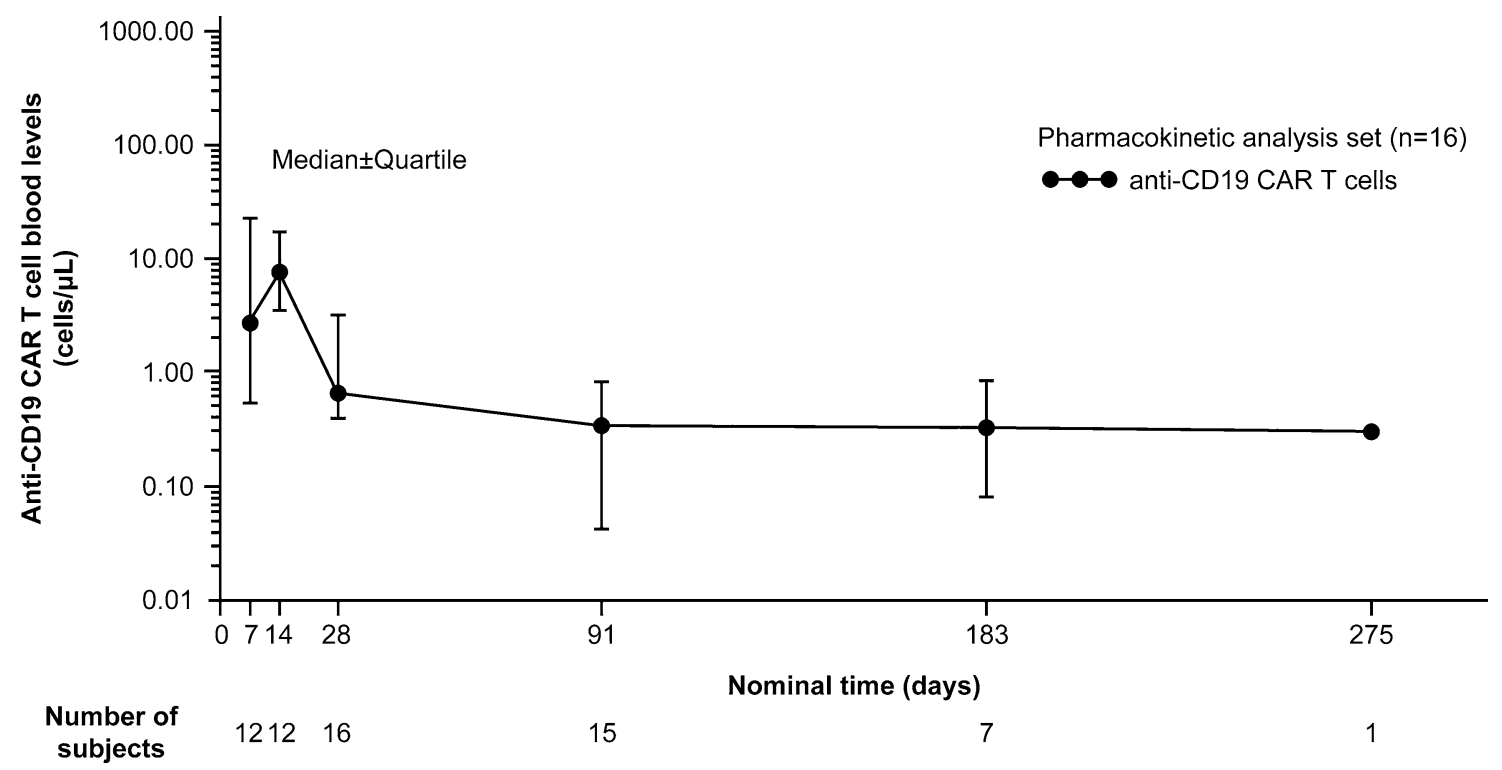

Fig. 4 Median blood level of anti-CD19 CAR T cells. CAR chimeric antigen receptor, $C D$ cluster of differentiation

Among the 17 patients who underwent leukapheresis, 16 received axi-cel infusion. The low incidence of discontinuation can be attributed to the short turn-around time.

One patient who relapsed after $\mathrm{CR}$ was re-administered with axi-cel, and CR was achieved again. No significant additional safety concerns of re-administration were raised. To evaluate the clinical value of axi-cel re-administration, further investigation is required.

Since there is no head-to-head study, it is difficult to compare the efficacy and safety of different CAR T therapies. In a matching adjusted indirect comparison of axi-cel and tisa-cel, it was suggested that axi-cel may have superior efficacy but a greater risk of grade 1 or 2 CRS [21]. Future real world data will further clarify the difference of CAR T therapies.

In conclusion, axi-cel demonstrated clinically meaningful efficacy and a manageable safety profile in Japanese patients with R/R large B-cell lymphoma. The ORR and incidence of TEAEs observed in Japanese patients were comparable with those observed in the ZUMA-1 study and the bridging was feasible. The short turn-around time and low dropout rate in this study are promising factors for the use of axi-cel. Thus, axi-cel can be a good treatment option for Japanese patients with R/R large B-cell lymphoma. Limitations of this study include the small sample size and short follow-up period. Long-term follow-up is ongoing to determine conversions of response over time and identify any late-onset TEAEs. Analysis of mechanisms of disease progressions is also underway. Axi-cel is being evaluated for earlier line usage in large B-cell lymphoma, as well as in indolent NHL (NCT03391466,
NCT03761056, NCT03105336) in the US and other parts of the world.

Supplementary Information The online version contains supplementary material available at https://doi.org/10.1007/s10147-021-02033-4.

Acknowledgements The authors thank all the patients, their families, and facility staff involved in this study. Medical writing support was provided by Sarayu Pai, PhD, of Cactus Life Sciences, Mumbai, India, and funded by Daiichi Sankyo Co. Ltd. Medical editing and publication management support was provided by Tomonari Yamashita and Daisuke Kuroki of Daiichi Sankyo Co., Ltd. We thank Sonia S. Jung, PhD, Jun Kawashima, MD, and Harry Miao, MD, PhD of Kite, a Gilead Company, for their invaluable advice. These data were presented in part at the 82nd Annual Meeting of the Japanese Society of Hematology, October 9-11, 2020 in Kyoto, Japan.

Funding This clinical trial was funded by Daiichi Sankyo Co., Ltd.

\section{Declarations}

Conflict of interest KK reports honoraria from Kyowa Kirin, Takeda, and MSD, research funds from Celgene, Daiichi Sankyo, Novartis, and Chugai, and scholarship donations from Mundipharma. JK reports research funding from Eisai. KS reports research findings from Kyowa Kirin, Otsuka Pharmaceutical, and Bristol-Myers Squibb, and scholarship donations from Nippon Shinyaku, Zenyaku Kogyo, Chugai, Kyowa Kirin, Takeda, and Eisai. KA reports honoraria from BristolMyers Squibb, Celgene, Janssen, and Novartis and research funds from Daiichi Sankyo. KI reports honoraria from Celgene, Novartis, Daiichi Sankyo, and Chugai and research funding from Celgene, Novartis, Daiichi Sankyo, and Chugai. TT reports honoraria from Celgene, Novartis, Bristol Myers Squibb, Janssen, and Takeda, research funds from Daiichi Sankyo, Novartis, Takara Bio, Janssen, and Takeda, and scholarship donations from Takeda and Bristol Myers Squibb. SS and KY are employees of Daiichi-Sankyo and report profit from stock from Daiichi Sankyo. KT reports honoraria from Daiichi Sankyo, Celgene, Takeda, HUYA Bioscience, Zenyaku Kogyo, Yakult, Chugai, Mun- 
dipharma, Eisai, and Ono. NU reports honoraria from CMIC, AbbVie, Daiichi Sankyo, Nippon Shinyaku, Celgene, Pfizer, and Astellas. KH reports honoraria from Takeda, Daiichi Sankyo, Towa Pharmaceutical, Meiji Seika Pharma, and Celgene, manuscript fees from Takeda, and scholarship donations from Eisai and Takeda. NF, TS, HS, and YK are employees of Daiichi Sankyo. The other authors have no conflict of interest.

Open Access This article is licensed under a Creative Commons Attribution 4.0 International License, which permits use, sharing, adaptation, distribution and reproduction in any medium or format, as long as you give appropriate credit to the original author(s) and the source, provide a link to the Creative Commons licence, and indicate if changes were made. The images or other third party material in this article are included in the article's Creative Commons licence, unless indicated otherwise in a credit line to the material. If material is not included in the article's Creative Commons licence and your intended use is not permitted by statutory regulation or exceeds the permitted use, you will need to obtain permission directly from the copyright holder. To view a copy of this licence, visit http://creativecommons.org/licenses/by/4.0/.

\section{References}

1. International Agency for Research on Cancer (2020) Japan Source: Globocan 2020. https://gco.iarc.fr/today/data/factsheets/ populations/392-japan-fact-sheets.pdf. Accessed 11 Feb 2021

2. Chihara D, Ito H, Matsuda T et al (2014) Differences in incidence and trends of haematological malignancies in Japan and the United States. Br J Haematol 164:536-545

3. Coiffier B, Sarkozy C (2016) Diffuse large B-cell lymphoma: R-CHOP failure-what to do? Hematology Am Soc Hematol Educ Program 2016(1):366-378

4. Crump M, Neelapu SS, Farooq U et al (2017) Outcomes in refractory diffuse large B-cell lymphoma: results from the international SCHOLAR-1 study. Blood 130:1800-1808

5. Makita S, Yoshimura K, Tobinai K (2017) Clinical development of anti-CD19 chimeric antigen receptor T-cell therapy for B-cell non-Hodgkin lymphoma. Cancer Sci 108:1109-1118

6. Neelapu SS, Locke FL, Bartlett NL et al (2017) Axicabtagene ciloleucel CAR T-cell therapy in refractory large B-cell lymphoma. N Engl J Med 377:2531-2544

7. Schuster SJ, Bishop MR, Tam CS et al (2019) Tisagenlecleucel in Adult Relapsed or Refractory Diffuse Large B-Cell Lymphoma. N Engl J Med 380:45-56

8. Abramson JS, Palomba ML, Gordon LI et al (2020) Lisocabtagene maraleucel for patients with relapsed or refractory large B-cell lymphomas (TRANSCEND NHL 001): a multicentre seamless design study. Lancet 396:839-852
9. Locke FL, Neelapu SS, Bartlett NL et al (2017) Phase 1 Results of ZUMA-1: a multicenter study of KTE-C19 anti-CD19 CAR T cell therapy in refractory aggressive lymphoma. Mol Ther 25:285-295

10. Locke FL, Ghobadi A, Jacobson CA et al (2019) Long-term safety and activity of axicabtagene ciloleucel in refractory large B-cell lymphoma (ZUMA-1): a single-arm, multicentre, phase 1-2 trial. Lancet Oncol 20:31-42

11. Jacobson C, Locke FL, Fhobadi A et al (2020) Long-Term Survival and Gradual Recovery of B Cells in Patients with Refractory Large B Cell Lymphoma Treated with Axicabtagene Ciloleucel (Axi-Cel). Blood 136(Supplement 1):40-42

12. Swerdlow SH, Campo E, Pileri SA et al (2016) The 2016 revision of the World Health Organization classification of lymphoid neoplasms. Blood 127:2375-2390

13. Cheson BD, Pfistner B, Juweid ME et al (2007) Revised response criteria for malignant lymphoma. J Clin Oncol 25:579-586

14. National Cancer Institute (2010) Common Terminology Criteria for Adverse Events (CTCAE) version 4.03. https://evs.nci.nih.gov/ ftp1/CTCAE/CTCAE_4.03/CTCAE_4.03_2010-06-14_Quick Reference_8.5x11.pdf. Accessed 8 Oct 2020

15. Lee DW, Gardner R, Porter DL et al (2014) Current concepts in the diagnosis and management of cytokine release syndrome. Blood 124:188-195

16. Mander AP, Thompson SG (2010) Two-stage designs optimal under the alternative hypothesis for phase II cancer clinical trials. Contemp Clin Trials 31:572-578

17. Locke FL, Rossi JM, Neelapu SS et al (2020) Tumor burden, inflammation, and product attributes determine outcomes of axicabtagene ciloleucel in large B-cell lymphoma. Blood Adv 4:4898-4911

18. Plaks V, Rossi JM, Chou J et al (2021) CD19 target evasion as a mechanism of relapse in large B-cell lymphoma treated with axicabtagene ciloleucel. Blood. https://doi.org/10.1182/blood.20210 10930

19. Neelapu SS (2019) Managing the toxicities of CAR T-cell therapy. Hematol Oncol 37(Suppl 1):48-52

20. Goto H, Makita S, Kato K et al (2020) Efficacy and safety of tisagenlecleucel in Japanese adult patients with relapsed/refractory diffuse large B-cell lymphoma. Int J Clin Oncol 25:1736-1743

21. Oluwole OO, Jansen JP, Lin VW et al (2020) Comparing efficacy, safety, and preinfusion period of axicabtagene ciloleucel versus tisagenlecleucel in relapsed/refractory large B cell lymphoma. Biol Blood Marrow Transplant 26:1581-1588

Publisher's Note Springer Nature remains neutral with regard to jurisdictional claims in published maps and institutional affiliations.

\section{Authors and Affiliations}

\section{Koji Kato ${ }^{1}$ (D) Shinichi Makita ${ }^{2} \cdot$ Hideki Goto $^{3} \cdot$ Junya Kanda $^{4} \cdot$ Nobuharu Fujii $^{5} \cdot K^{2}$ azuyuki Shimada ${ }^{6}$ Koichi Akashi $^{1}$. Koji Izutsu $^{2} \cdot$ Takanori Teshima $^{3} \cdot$ Natsuko Fukuda $^{7} \cdot$ Tokuhito Sumitani $^{7} \cdot$ Hiroyuki Sumi $^{7} \cdot$ Shinji Shimizu $^{7}$. Yasuyuki Kakurai $^{7} \cdot$ Kenji Yoshikawa $^{7} \cdot$ Kensei Tobinai $^{8} \cdot$ Noriko Usui $^{9} \cdot$ Kiyohiko Hatake $^{10}$}

1 Department of Medicine and Biosystemic Science, Kyushu University Graduate School of Medical Sciences, 3-1-1, Maidashi, Fukuoka Higashi-ku, Fukuoka 812-8582, Japan

2 Department of Hematology, National Cancer Center Hospital, 5-1-1, Tsukiji , Chuo, Tokyo 104-0045, Japan
3 Department of Hematology, Hokkaido University Faculty of Medicine, Kita 15, Nishi 7, Kita-ku, Sapporo 060-8638, Japan 
4 Department of Hematology and Oncology, Graduate School of Medicine, Kyoto University, Yoshida-Konoe-cho, Sakyo-ku, Kyoto 606-8501, Japan

5 Department of Hematology and Oncology, Okayama University Hospital, 2-5-1 Shikata-cho, Kitaku,

Okayama 700-8558, Japan

6 Department of Hematology and Oncology, Nagoya University Graduate School of Medicine, 65 Tsurumai-cho, Showa-ku, Nagoya 466-8550, Japan

7 Daiichi Sankyo Co., Ltd, 1-2-58, Hiromachi, Shinagawa, Tokyo 140-8710, Japan
8 Geriatric Health Services Facility Rehabilitation Care Funabashi, 4-8-30 Honcho, Funabashi, Chiba 273-005, Japan

9 Department of Clinical Oncology and Hematology, The Jikei University Daisan Hospital, 4-11-1 Izumihoncho, Komae, Tokyo 201-8601, Japan

10 Department of Lymphoma/Hematology Center, Mita Hospital, International University of Health and Welfare, 1-4-3 Mita, Minato, Tokyo 108-8329, Japan 